\title{
EVALUACIÓN DE TÉCNICAS PARA EL MUESTREO DE ORGANISMOS BENTÓNICOS: CENSOS VISUALES vS SURBER
}

\author{
Alberto Javier Perán Rex ${ }^{1}$, Josefa Velasco García, Andrés Millán Sánchez \\ Departamento de Ecología e Hidrología, Facultad de Biología, Universidad de Murcia, Campus de Espinardo, \\ 30 100, Murcia, España. - inland@Wanadoo
}

\section{RESUMEN}

Como alternativa a los métodos de captura tradicionales de muestreo de organismos acuáticos bentónicos se propone la utilización de técnicas visuales mediante la obtención de imágenes fotográficas o de vídeo. Para testar la utilidad de esta técnica se realizó un muestreo mediante fotografía de 17 unidades de $35 \mathrm{~cm}^{2}$ de superficie sobre una población de larvas de simúlidos en el río Chícamo (Cuenca del Río Segura, SE Ibérico). Las imágenes fueron procesadas usando el programa UTHSCSA Image Tool de distribución gratuita (ftp://maxrad6.uthscsa.edu). Los resultados fueron comparados con los obtenidos de forma simultánea mediante la toma del mismo número de muestras con un Surber de igual superficie.

Se encontraron diferencias significativas en el número total de larvas de simúlidos censadas entre ambos métodos y en algunas clases de tamaño. La técnica visual empleada comparada con el Surber constituye un método más preciso ya que se evita la pérdida de individuos que ocurre normalmente con los métodos tradicionales de cáptura. Además, la técnica visual resulta más eficaz pues requiere un menor número de muestras para estimar la densidad de la población con un mismo error (24 muestras frente a 27 , para un error del $20 \%$ de la media). La abundancia de larvas de las clases de tamaño más pequeñas (1-5 mm) fue significativamente mayor en los censos visuales que en la obtenida con Surber, mientras que para las clases de tamaño superiores $(6-8 \mathrm{~mm})$ no se encontraron diferencias significativas. Los censos visuales se mostraron, por tanto, más efectivos y precisos para estudiar poblaciones de larvas de simúlidos y pueden ser Útiles para el estudio de otros grupos de invertebrados acuáticos bentónicos, como Gasterópodos y Tricópteros, en ambientes someros, de aguas claras y de baja turbulencia y con sustrato relativamente homogéneo. Su aplicabilidad a otros grupos de organismos de mayor movilidad o menos visibles es discutida.

Palabras clave: fotografía, censos visuales, métodos muestreo, bentos, simúlidos, ríos, análisis automático de imagen.

\begin{abstract}
Wepropose the use of photography or video images as an alternative to traditional sampling methods of capture of benthic aquatic organisms. To test the utility of this technique $I 7$ units of $35 \mathrm{~cm}^{2}$ were randomly selected and photographed from a population of simulid larvi in the Stream Chícamo (River Segura Basin, SE of the Iberian Peninsula). The images were processed using the program UTHSCSA Image Tool of free distribution ([ftp]:// maxrad6.uthscsa.edu). Results were compared with those obtained by simultaneously sampling with a Surber of equal surface.

Wefound significant differences between both methods in the total number of simulid larvi and in the abundance of some size classes. The visual technique used was more accurate than Surber in the estimation of real abundance of the population, because it prevented the loss of individuals caused by traditional methods of capture. Besides, the visual technique requires less number of samples than Surber to estimate the density of the population with the sume accuracy (24and 27 samples, respectively, for a $20 \%$ error). The abundance of larvae of the smaller size classes $(1-5 \mathrm{~mm})$ was significantly higher in the visual census than in those obtained with Surber, while for the higher size classes $(6-8 \mathrm{~mm})$ we did not find significant differences. As a conclusion, the visual technique is more effective in the study of populations of simulid larvi. The new method could be usefulfor the study of other groups of benthic aquatic invertebrates, such as Gastropoda and Trichoptera, in shallow and clear waters, in places with little turbulence flow and a homogeneous substrate. Its applicability to more mobile or more criptic organisms is also discussed.
\end{abstract}

Key words: photography, visual census, sampling methods, benthos, Simulidae, streams, image analysis. 


\section{INTRODUCCIÓN}

La elección del método de muestreo es una de las decisiones más importantes a la hora de abordar el estudio de organismos. Puesto que la mayoría de los métodos de captura no consiguen obtener valores absolutos de la abundancia de la población o comunidad, sino que exhiben cierto error, los investigadores llevan décadas esforzándose en diseños instrumentales que reduzcan al mínimo dicho error. De esta forma se han desarrollado multitud de métodos de muestreo, más o menos complejos, y a menudo, específicamente adaptados a los diversos tipos de hábitat existentes en las aguas continentales (Rosenberg, 1978; Merrit et al 1978; Elliot \& Tullet, 1983; Boulton, 1985; Brooks, 1994). Un número equivalente de artículos ha testado y comparado la fiabilidad de estos métodos (Crossman \& Cairns, 1974; Meehan \& Elliot, 1974; Mackie \& Bailey, 1981; Chadwick \& Canton, 1983; Brinkman \& Duffy, 1996; Humphries et al., 1998). La selección de la técnica de muestreo se ha convertido en un complicado arte que intenta optimizar la exactitud y la precisión de las estimas, con el coste tanto en tiempo como en dinero y la facilidad de manejo. Este compromiso adquiere su máxima expresión en los muestreos intensivos necesarios para el estudio de los ciclos de vida y producción secundaria, donde el investigador ha de realizar numerosas estimas de la población bajo estudio. Esta circunstancia unida a la necesidad adicional de contar y medir cada individuo supone un esfuerzo tan considerable que ha hecho desistir a muchos investigadores de acometer este tipo de estudios (Benke, 1984).

Aunque los censos visuales ya han sido utilizados ampliamente en otros campos como la biología marina (Torlegard \& Lundalv, 1974; Lundalv, 1971, 1985; Lundalv et al. 1986) hasta el momento no se ha desarrollado una metodología aplicable a organismos de aguas continentales. En el presente artículo se evalúa la efectividad de los censos visuales en la estima de la abundancia de una población de larvas de simúlidos (Diptera) frente al Surber, uno de los métodos tradicionales de captura más utilizado. Final- mente se discuten las ventajas e inconvenientes del método y su aplicabilidad al muestreo de otros grupos de organismos bentónicos.

\section{MATERIAL Y MÉTODOS}

\section{Área de estudio}

El estudio se realizó sobre una población de Simulium (Eusimulium) cf. angustipes en un tramo permanente de unos 100 metros de longitud en el río Chícamo, un afluente hiposalino del río Segura en el SE de la Península Ibérica. Se trata de un arroyo espacialmente intermitente que drena una cuenca de $502 \mathrm{~km}^{2}$. La longitud total del cauce principal es de $59.4 \mathrm{~km}$, pero de ellos

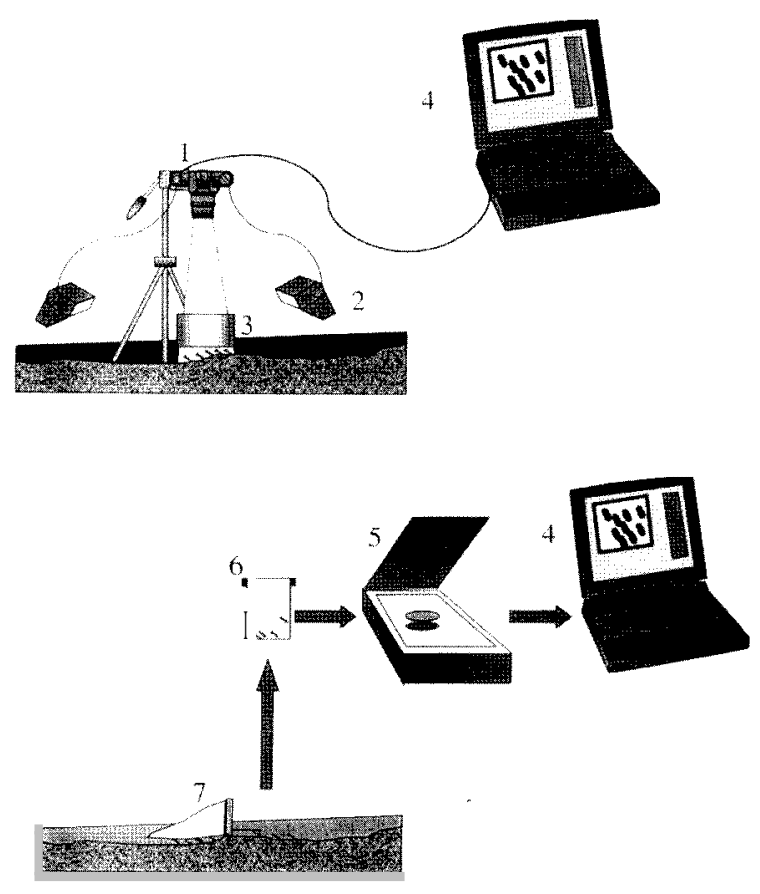

Figura 1. Equipamiento necesario para el muestreo de larvas de simúlidos por los dos métodos utilizados. a) censo visual: b) Surber. 1.- cámara fotagráfica; 2.- flash; 3.- placa Petri; 4.- ordenador: 5.escáner; 6.- muestra; 7.-Surber. Equipment required to sampling simulid larvi by both methods used. a) visual census; b) Surber. 1.camera; 2.- flash; 3.- Petri dish: 4.- computer: 5.- scanner; 6.- sample; 7.- Surber. 
sólo $22 \mathrm{~km}$ presentan un flujo permanente, aunque separados por tramos donde el flujo superficial está restringido a los episodios lluviosos. El caudal medio anual es de $4.28 \mathrm{l} / \mathrm{s}$ y la profundidad del agua raramente supera los $5 \mathrm{~cm}$. El lecho, constituido de margas compactadas, se estructura en pequeñas pozas y rápidos. Es en las zonas de rápidos donde se concentra la población bajo estudio, siendo perfectamente visible la distribución agregada de sus individuos, dada la transparencia del agua.

\section{Muestreo y procesado de los individuos}

Con el fin de evaluar la eficacia de los censos visuales se realizó un muestreo estratificado y al azar de la población de simúlidos en las zonas de corriente utilizando primero la toma fotográfica de un agregado de individuos y después la toma de muestra mediante Surber de $35 \mathrm{~cm}^{2}$ de superficie sobre dicho agregado. En total se muestrearon 17 agregados, resultando el mismo número de muestras para ambos métodos. La técnica fotográfica empleada, consiste en la fotografia de una cuadrícula de $60 \mathrm{~cm}^{2}$ de superficie con una cámara fotográfica Nikon 301 y objetivo de 35 $\mathrm{mm}$, provista de un trípode $\mathrm{y}$, opcionalmente, con flashes de relleno para evitar la formación de sombras (Fig. 1). Como las turbulencias del agua a menudo distorsionaban la imagen, una simple placa Petri dispuesta de forma invertida en la superficie del agua y de manera que no disminuyera el flujo de agua, eliminaba dicho efecto. La ubicación en el lecho del río de una regla permitió el posterior calibrado de la imagen. Las imágenes así obtenidas fueron digitalizadas y procesadas a través del analizador de imagen Image Tool 1.27 (desarrollado por el Centro de Estudios de la Salud de la Universidad de San Antonio, Texas y disponible de forma gratuita a través de internet por FTP anónimo en la dirección $\mathrm{ftp}: / /$ maxrad6.uthscsa.edu), con el que, tras la calibración de cada toma y selección de una cuadrícula aleatoria de $7 \times 5 \mathrm{~cm}$, se contaron y midieron las longitudes de cada uno de los individuos hasta $0.1 \mathrm{~mm}$ de precisión. Los individuos medi- dos fueron agrupados en clases de tamaño de 1 mm para su posterior análisis.

Las muestras fueron conservadas en alcohol al $70 \%$ y posteriormente procesadas en el laboratorio para la separación de los individuos. Finalmente, las imágenes de los ejemplares fueron capturadas en un escáner y procesadas de idéntica forma al caso anterior, evitando así los errores debidos al método de medida empleado. Dicho procesado de las muestras se realizó lo más rapidamente posible para evitar al máximo las alteraciones de tamaño que los líquidos fijadores ocasionan.

\section{Análisis de los datos}

A partir de los datos de abundancia obtenidos con cada uno de los métodos de muestreo, se calculó el tamaño muestral necesario para obtener una estima fiable de la población con un error estándar de la media del 20\%. Para ello se utilizó el método recomendado por Elliot (1971) para poblaciones con distribución contagiosa y cuando el número de muestras es inferior a 30 . Para comparar ambos métodos, se utilizó el análisis de la varianza (ANOVA, un factor) tanto para el número total de larvas recogidas por muestra como para la abundancia de cada clase de tamaño. Los datos originales fueron transformados logarítmicamente $(\log (x+1))$ con el fin de normalizar su distribución.

\section{RESULTADOS}

En la Tabla 1 aparecen para cada uno de los métodos de muestreo utilizados, la abundancia de las diferentes clases de tamaño y el número total de individuos recogidos en las 17 muestras así como los estadísticos descriptivos básicos. El número total de organismos registrados fué de 659 individuos para el muestreador Surber y de 1171 individuos para el censo visual. Como se puede observar, la abundancia media de la población obtenida mediante la técnica del censo visual es casi el doble que la obtenida con el Surber, aun- 
Tabla 1. Estadistícos descriptivos, abundancia total y abundancia de cada una de las clases de tamaño de las larvas de Simulium (Eusimulium) cf. angustipes en las 17 muestras obtenidas por ambos métodos de muestreo. ES, es el error estandar de la media. Descriptive statistics, total abundance and class abundance of larvae of Simulium (Eusimulium) cf. angusiipes in 17 samples obtained by both methods. ES is the standar error of the mean.

MUESTREO VISUAL (n=17)

\begin{tabular}{|c|c|c|c|c|c|c|c|c|c|c|c|c|c|c|c|c|c|c|c|c|c|}
\hline Clase & 1 & 2 & 3 & 4 & 5 & 6 & 7 & 8 & 9 & 10 & 11 & 12 & 13 & 14 & 15 & 16 & 17 & $\mathbf{N}$ total & Media & Desvstd. & ES \\
\hline $0-1 \mathrm{~mm}$ & 1 & 0 & 0 & 1 & 0 & 0 & 0 & 1 & 0 & 0 & 0 & 0 & 0 & 2 & 0 & 1 & 0 & 6 & 0.35 & 0.61 & 0.15 \\
\hline $1-2 m m$ & 11 & 8 & 1 & 16 & 0 & 9 & 10 & 16 & 19 & 3 & 0 & 0 & 3 & 4 & 2 & 13 & 0 & 115 & 6.76 & 6.47 & 1.57 \\
\hline $2-3 \mathrm{~mm}$ & 58 & 27 & 6 & 23 & 7 & 67 & 13 & 13 & 42 & 6 & 14 & 3 & 11 & 9 & 2 & 20 & 6 & 327 & 19.24 & 19.19 & 4.65 \\
\hline $3-4 \mathrm{~mm}$ & 42 & 17 & 8 & 18 & 11 & 39 & 31 & 30 & 23 & 30 & 9 & 18 & 8 & 7 & 5 & 29 & 4 & 329 & 19.35 & 12.26 & 2.97 \\
\hline $4-5 \mathrm{~mm}$ & 23 & 7 & 4 & 27 & 17 & 46 & 29 & 13 & 10 & 26 & 10 & 16 & 9 & 4 & 1 & 25 & 3 & 270 & 15.88 & 12.03 & 2.92 \\
\hline $5-6 \mathrm{~mm}$ & 6 & 0 & 08 & 1 & 7 & $1 \varepsilon$ & $8 \quad 1$ & 0 & 23 & 9 & 2 & 1 & 1 & 1 & 0 & 7 & 2 & 96 & 5.65 & 5.83 & 1.41 \\
\hline $6-7 \mathrm{~mm}$ & 1 & 0 & 0 & 0 & 6 & 2 & 1 & $\mathrm{O}$ & 0 & 2 & 0 & 1 & 0 & 0 & 0 & 0 & 0 & 13 & 0.76 & 1.52 & 0.37 \\
\hline $7-8 \mathrm{~mm}$ & 0 & 0 & 0 & 0 & 1 & 0 & 0 & 0 & 0 & 1 & 0 & 0 & 0 & 0 & 0 & 0 & 0 & 2 & 0.12 & 0.33 & 0.08 \\
\hline total & 142 & 59 & 19 & 93 & 59 & 181 & 94 & 75 & 97 & 77 & 35 & 49 & 32 & 26 & 10 & 95 & 15 & 1158 & 68.12 & 46.43 & 11.26 \\
\hline
\end{tabular}

MUESTREO CON SURBER $(n=17)$

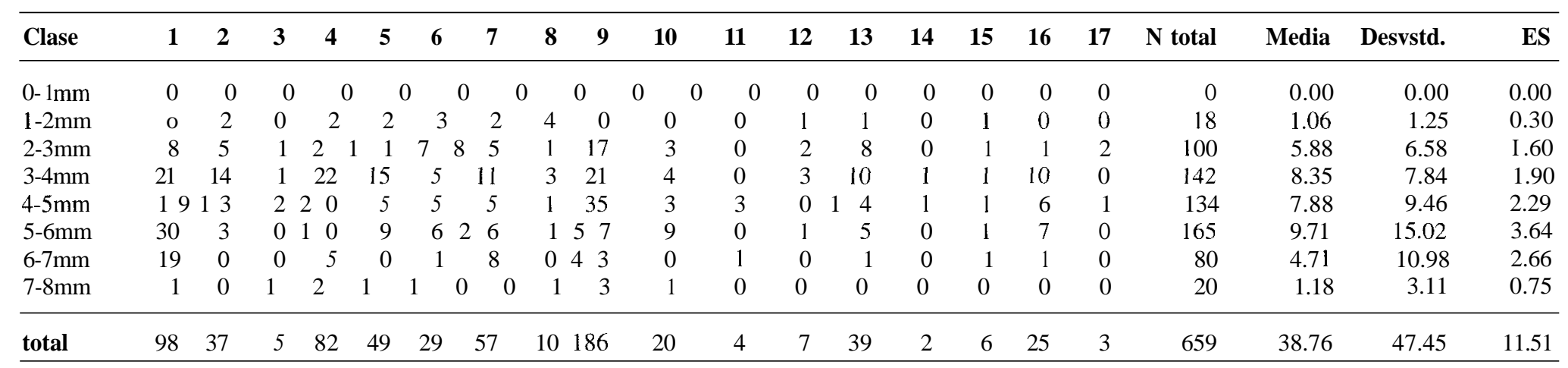




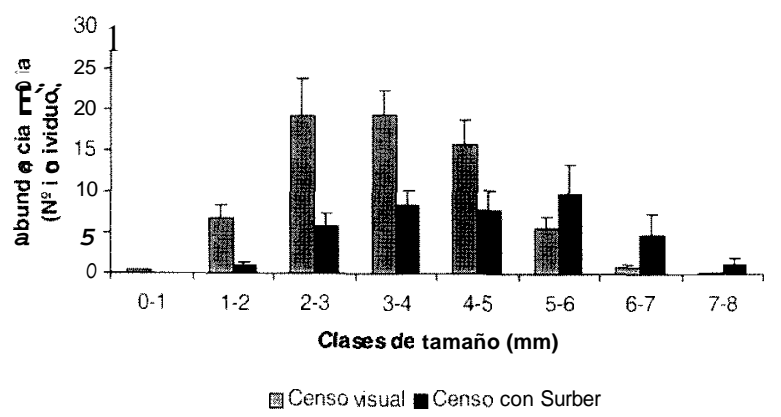

Figura 2. Distribución de las abundancias medias de las clases de tamaño de las larvas de simúlidos obtenidas por ambos métodos de muestreo. Distribution of mean abundance distribution of simulids larvue size classes obtainedfrom both sampling methods.

que se presenta una desviación típica muy similar entre las muestras para ambos métodos. Se han encontrado diferencias significativas en la abundancia total de larvas de simúlidos entre ambos métodos $(\mathrm{F}=7.164, \mathrm{p} \leq 0.0116)$.

Por otra parte, el número de muestras necesario para estimar la abundancia de la población con un error del $20 \%$ de la media fue de 24 muestras para la técnica fotográfica frente a 27 muestras para el muestreo con Surber.

Analizando la distribución de abundancias medias de las clases de tamaño obtenida por ambos métodos (Fig. 2), se observa que las clases de tamaño intermedias fueron las más abundantes, aunque se registraron mayores valores con

Tabla 2. Resultados de los análisis de la varianza efectuados para las abundancias de cada una de las clases de tamaño. Analysis of variance results applied to the abundunce of each size class.

\begin{tabular}{lrc}
\hline ANOVAS & F & P \\
\hline Clase I $(0-1 \mathrm{~mm})$ & 6.276 & 0.017 \\
Clase 11(1-2mm) & 11.272 & 0.002 \\
Clase III (2-3 mm) & 12.602 & 0.001 \\
Clase IV (3-4mm) & 10.992 & 0.002 \\
Clase V (4-5mm) & 6.771 & 0.014 \\
Clase VI (5-6mm) & 0.127 & 0.723 \\
Clase VII $(6-7 \mathrm{~mm})$ & 2.223 & 0.146 \\
Clase VIII $(7-8 \mathrm{~mm})$ & 3.805 & 0.060 \\
\hline
\end{tabular}

los censos visuales. Las clases más pequeñas (I y II), aunque menos abundantes, también presentaron mayores abundancias medias en los censos visuales que en las muestras obtenidas con Surber. Sin embargo, los datos de abundancia de las clases más grandes (VI, VII y VIII) fueron más altos para el muestreo con Surber que con el censo visual. Dichas diferencias en la abundancia fueron significativas para las clases de tamaño I,II, III, IV y V, mientras que para el resto de clases (VI, VII y VIII), no se encontraron diferencias significativas entre ambos métodos (Tabla 2).

\section{DISCUSIÓN}

En vista a los resultados obtenidos, los censos visuales presentan una precisión y eficacia de muestreo mayor que el muestreador Surber ya que se evita la pérdida y deterioro de organismos, fundamentalmente los de menor tamaño, que ocurre con los métodos tradicionales de captura. La pérdida de sedimentos y organismos por el borde inferior del Surber y los huecos que quedan con el sustrato ha sido puesta de manifiesto por varios autores (Kroger, 1972; Boulton, 1985). Kroger (1972) hace referencia a pérdidas superiores al 75\% con el Surber. En el presente estudio, las pérdidas respecto a los censos visuales fueron del $43 \%$, y eso a pesar de que los muestreos se realizaron sobre superficies bastante planas y por tanto idóneas para el Surber. En cambio, los censos visuales al no implicar la separación de los individuos del sustrato, no ocasionan la pérdida de ninguno de ellos. Además no requieren la fijación y separación de los individuos de la muestra que normalmente conllevan una gran parte del tiempo del estudio. Esto supone un ahorro importante de tiempo y medios, que se puede invertir en aumentar el número de muestras y la precisión de las estimas. Por otra parte al no implicar la fijación y conservación de los organismos se evitan las alteraciones morfológicas que los líquidos fijadores ocasionan, como cambios en forma, tamaño y peso (Donald \& Paterson, 1977; Leslie \& Moore, 1986; Nolte, 1990). 
Otra ventaja adicional del método es que permite realizar muestreos intensivos sobre poblaciones no muy abundantes sin afectar su abundancia y dinámica poblacional, lo que resulta de gran interés en el caso de estudios de ciclos de vida o producción secundaria.

Los censos visuales se mostraron, por tanto, más precisos y menos costosos (exceptuando el coste inicial del equipo fotográfico) que el Surber a la hora de estudiar este tipo de poblaciones bentónicas. Su aplicabilidad a otros grupos de organismos acuáticos aun no se ha testado, pero pueden ser útiles en el muestreo de organismos bentónicos de superfície, como Gasterópodos, Tricópteros, etc. de escasa movilidad y fuerte contraste con el sustrato. Sin embargo, para organismos que viven bajo piedras (ej: larvas de Efemerópteros y Plecópteros) o entre los sedimentos (ej: larvas de Quironómidos), la técnica fotográfica no sería aplicable directamente.

Por otra parte, las técnicas visuales, especialmente el vídeo, pueden ser útiles en el muestreo de organismos nadadores, como muchas especies de Heterópteros y Coleópteros acuáticos. Dichos organismos presentan reacciones de huida ante cualquier pequeña modificación del medio (cambios de luz, turbulencias, etc.). En estas circunstancias los censos visuales presentarían la significativa ventaja de posibilitar la operación a distancia ya que tras situar la cámara sobre la zona de muestreo es posible accionarla de forma remota transcurrido el tiempo necesario para el retorno de los individuos huidos, y obtener tomas a intervalos fijos de tiempo. Otra posibilidad de las técnicas visuales es su capacidad de obtener de forma simultánea a la medición, las coordenadas espaciales de cada individuo, lo que permitiría detallados análisis de la distribución espacial de los organismos y de sus trayectorias.

Indiscutiblemente las técnicas visuales presentan fuertes limitaciones a la hora de su aplicación como son la visibilidad y contraste de los organismos sobre el fondo y que las aguas sean transparentes y poco turbulentas. Estas condiciones son comunes en pequeños arroyos de cabecera o en cursos medios de ríos de zonas áridas y semiáridas como el estudiado. Por otro lado. en situa- ciones de grandes densidades de organismos en las que se produzca la superposición de unos sobre otros, no son posibles medidas morfométricas fiables, aunque sí recuentos.

En resumen, los censos visuales, cuando sea posible su utilización, pueden resultar una ventajosa alternativa de muestreo.

\section{BIBLIOGRAFIA}

BENKE, A.C. 1984. Secondary production of aquatic insects. In: The Ecology of Aquatic Insects. V.H. Resh \& D.M. Rosemberg (eds.): 219-232. Praeger Publishers, New York, USA.

BOULTON, A.J. 1985. A sampling device that quantitatively collects benthos in flowing or standing waters. Hydrobiologia, 127:31-39.

BRINKMAN, M.A. \& W.G. DUFFY. 1996. Evaluation of four wetland aquatic invertebrate samplers and four sampling sorting methods. $J$. Freshwat. Ecol., 11(2): 192-200.

BROOKS, S. 1994. An efficient and quantitative aquatic benthos sampler for use in diverse habitats with variable flow regimes. Hydrobiologia, 281: 123-128.

CROSSMAN, J.S. \& J. Jr. CAIRNS, 1974. A comparative study between two artificial substrate samplers and regular sampling techniques. Hydrobiologia, 44: 517-522.

CHADWICK, J.W. \& S.P. CANTON, 1983. Comparison of multiplate and Surber samplers in a Colorado mountain stream. J. Freshwat. Ecol., 2: 287-292.

DONALD, G.L. \& C.G. PATERSON. 1977. Effect of preservation on wet biomass of chironomid larvae. Hydrobiologia, 53: 75-80.

ELLIOT, J.M. 1971. Some methodsfor the statistical analysis of samples of benthic invertebrates. Freshwat. Biol. Ass. Scientific Publication no 25.

ELLIOT, J.M. \& P.A.TULLET. 1983.A supplement to a bibliography of samplers for benthic invertebrates. Oec. Publ. Freshwat. Biol. Ass. n 20, 27pp.

HUMPHRIES, P; GROWNS, J.E.; SERAFINI, L.G.; HAWKING; J.H:; CHICK, A.J. \& P.S. LAKE. 1998. Macroinvertebrate sampling methods for lowland Australian rivers. Hydrobiologia, 364: 209-218.

KROGER, R.L.1972. Underestimation of standing crop by the Surber sampler. Limnol. Oceanogr., 17: $475-478$. 
LESLIE, J.K. \& J.E. MOORE. 1986. Changes in lengths of fixed and preserved young freshwater fish. Can. J. Aquat. Sci., 43: 1079-1081.

LUNDALV, T. 1971. Quantitative studies on rockybottom bioceonoses by underwater photogrammetry. A methodological study. Thalassia Jugosl., 7: 201-208.

LUNDALV, T. 1985. Detection of long-term trends in rocky sublitoral communities: Representativeness of fixed sites. In: The ecology of rocky coasts. P.G. Moore \& R. Seed (eds.): 329-345. Hodder and Stoughton Educational, U.K.

LUNDALV, T.,C. LARSSON \& L. AXELSON. 1986. Long-term trends in algal-dominated rocky subtidal communities on the Sedish west coast - a transitional system? Hydrobiologiu., 142: 81-95.

MACKIE, G.L. \& R.C. BAILEY, 1981.An inexpensive stream bottom sampler. J. Frewshwat. Ecol., 1: 61-69.
MEEHAN, W.R. \& S.T. ELLIOT. 1974. Comparative effectiveness of the standard Surbers sampler and a hydraulic modification for estimating bottom fauna populations. Prog. Fish. Cult., 36: 16-19.

MERRIT, R.W.; CUMMINS, K.W. \& V.H.L. RESH. 1978. Collecting, sampling and rearing methods for aquatic insects. In: An introduction to aquatic insects of North America. R.W. Merrit \& K.W. Cummins (eds.): 13-28. Kendall-Hunt Publishing Co., Dubuque, Iowa, USA.

NOLTE, U. 1990. Chironomid biomass determination from larval shape. Freshwut. Biol., 24: 443-451.

ROSENBERG, D.M., 1978. Practical sampling of freshwater macrozoobenthos: A bibliography of useful texts, reviews, and recent papers. Can. Fish. Mur: Serv. Tech. Rep., 790, 15 pp.

TORLEGARD, A.K.I. \& LUNDALV,T. 1974. Underwater analytical system. Photogramm. Eng., 40: 287-293. 